\title{
Use of Anticoagulants in Patients with Pulmonary Hypertension
}

\author{
Laurent Bertoletti ${ }^{1,2,3,4}$ Valentine Mismetti ${ }^{1,5}$ George Giannakoulas ${ }^{6}$
}

\author{
${ }^{1}$ Service de Médecine Vasculaire et Thérapeutique, $\mathrm{CHU}$ de \\ St-Etienne, Saint-Etienne, France \\ 2 INSERM, UMR1059, EquipeDysfonctionVasculaire et Hémostase, \\ Université Jean-Monnet, Saint-Etienne, France \\ 3 INSERM, CIC-1408, CHU Saint-Etienne, Saint-Etienne, France \\ ${ }^{4}$ F-CRIN INNOVTE Network, Saint-Etienne, France \\ ${ }^{5}$ Service de Pneumologie, CHU de St-Etienne, St-Etienne, France \\ 6 Department of Cardiology, Aristotle University of Thessaloniki, \\ AHEPA Hospital, Thessaloniki, Greece
}

Hämostaseologie 2020;40:348-355.
Address for correspondence Laurent Bertoletti, MD, PhD, Service de MédecineVasculaire et Thérapeutique, CHU de St-Etienne, F-42055, Saint-Etienne, France (e-mail: laurent.bertoletti@gmail.com).

\begin{abstract}
Keywords

- anticoagulant

- pulmonary hypertension

- venous thromboembolism

- bleeding

Since the earliest works on the understanding of different forms of pulmonary hypertension, thrombosis has been involved in the pathophysiology of the disease, both in pulmonary arterial hypertension (PAH) and in chronic thromboembolic pulmonary hypertension (CTEPH). Autopsy and then pathophysiological data paved the way for the use of anticoagulants as a treatment for PAH and CTEPH. In PAH their role has diminished with the advent of specific targeted therapies, but they are still prescribed in more than half of PAH patients, because of concomitant venous thromboembolism or atrial fibrillation. In CTEPH long-term anticoagulant therapy is the cornerstone of the management. The recent development of direct oral anticoagulants (DOACs) raises the question of the best anticoagulation strategy, both in patients with PAH and in patients with $\mathrm{CTEPH}$. In this review, we present an overview of the history of anticoagulants in the management of patients suffering from PAH or CTEPH, an update of the available data on the underlying rationale of their use in these subjects, an alert on the potential risks of using DOACs in these poorly explored situations, and the setting up of dedicated trials to evaluate the best anticoagulant treatment strategies in patients suffering from PAH or CTEPH.
\end{abstract}

\section{Introduction}

Pulmonary hypertension (PH) is defined by an abnormal elevation of mean pulmonary arterial pressure. ${ }^{1}$ It can occur in different situations, either autonomously (as in pulmonary arterial hypertension [PAH], which may be associated with others conditions), or as a complication of other cardiac or respiratory clinical entities: left heart diseases (postcapillary $\mathrm{PH}$ ), pulmonary embolism (PE; chronic thromboembolic PH, CTEPH), chronic lung diseases, and/or hypoxia. Thus, there is an international clinical classification of PH, separating precapillary (group 1, 3, 4, and 5) from postcapillary causes (group 2). ${ }^{2}$ In every situation, the development of $\mathrm{PH}$ is associated with a worse prognosis, by the development of right heart failure as the main cause of death.

During the past decade, significant improvements have been achieved in patients with group 1 (PAH) and group 4 (CTEPH). Historically, PAH has aroused the greatest interest, particularly after iatrogenic causes had been identified, such as anorexigens. ${ }^{3}$ PAH may be idiopathic, heritable, drug- and toxin- induced, or associated with conditions such as connective tissue diseases (CTD-PAH). Several targeted therapies for PAH have been developed in the past two decades. ${ }^{4}$ Before the advent of these drugs, treatment of PAH was mainly based on diuretics, oxygen, and oral anticoagulants. This conventional therapy remains important as the initial received

January 24, 2020

accepted after revision

May 5, 2020 (c) 2020 Georg Thieme Verlag KG Stuttgart · New York
DOI https://doi.org/

10.1055/a-1171-3995.

ISSN 0720-9355. 
step of the treatment algorithm of patients with $\mathrm{PAH} .^{5}$ During the same time, there have been two major advances in the treatment of CTEPH, with the development of medical and interventional treatment, in patients ineligible to surgery (pulmonary endarterectomy), which is the treatment of choice to relieve pulmonary artery obstructions when possible. However, whatever the treatment option retained for a CTEPH patient, lifelong anticoagulation therapy remains the cornerstone of medical therapy.

In parallel with these advances in the management of PAH and CTEPH, anticoagulant treatment modalities in venous thromboembolism (VTE) and atrial fibrillation (AF) have been profoundly modified with the emergence of direct oral anticoagulants (DOACs), gradually replacing vitamin $\mathrm{K}$ antagonists (VKAs) in most of their indications. ${ }^{6}$ The arrival of these drugs, as well as their pharmacological particularities, justifies the need to redefine the role of anticoagulants in the treatment of patients suffering from $\mathrm{PAH}$ or CTEPH.

\section{General Review and Clinical Implications}

\section{Why Is Anticoagulant Therapy Prescribed to Patients with Pulmonary Arterial Hypertension or Chronic Thromboembolic Pulmonary Hypertension?}

\section{Patients with PAH}

\section{Rationale for Treating PAH with Warfarin}

From a pathophysiological point of view, PAH is characterized by the association of lesions in the distal muscular-type arteries: medial hypertrophy/hyperplasia, intimal and adventitial fibrosis, and (in situ) thrombotic lesions/plexiform lesions. ${ }^{7}$ The frequency of thrombotic lesions in autopsy studies, ${ }^{8}$ and the demonstration of both an associated activation of the coagulation system ${ }^{9-12}$ and a decreased in physiological fibrinolysis, ${ }^{13}$ paved the way for the rationale of use of anticoagulant drugs for the treatment of PAH. These hypotheses were comforted by the sub-analysis of a landmark trial evaluating the use of calcium blockers in patients with $\mathrm{PAH},{ }^{14}$ in which a better prognosis was described for patients who received warfarin. A summary of this study and of other prospective trials regarding the efficacy and safety of anticoagulant therapy in patients with $\mathrm{PAH}$ is provided in -Table 1. Of note, the allocation of warfarin was not provided by the randomization, but was advised to the investigators, in the case of the presence of an abnormal perfusion lung scan. Then, anticoagulant therapy with warfarin was prescribed in the majority of patients with $\mathrm{PAH}$, for a potential benefit on survival despite the absence of clear validation. ${ }^{15-17}$ This may explain the high prevalence of anticoagulant therapy ( $\sim 90 \%)$ in the epidemiological studies from the current era. ${ }^{18}$

In parallel, some studies indicated that patients with $\mathrm{PAH}$ experience a dysfunction of platelets, ${ }^{19,20}$ suggesting a potential place for antiplatelets in the treatment of PAH. This hypothesis was the primary aim of a randomized controlled trial ( - Table 1), evaluating aspirin (and simvastatin, in a crossover design) efficacy in patients with $\mathrm{PAH} .{ }^{21}$ Although aspirin confirmed its efficacy on a surrogate endpoint (level of thromboxane B2), it failed to demonstrate any effect on the 6-minute walk distance (which was the main endpoint for any PAH-targeted therapy at that time).

Concomitant Validated Indication for Anticoagulant Therapy The phenotype of patients diagnosed with $\mathrm{PAH}$ changed deeply in the past two decades. ${ }^{22}$ While patients included in the historic premodernmanagement National Institutes of Health registry were most frequently young females, ${ }^{23}$ current patients included in contemporary registries are older and with a more balanced sex ratio at time of PAH diagnosis. ${ }^{24-26}$ These moving phenotypes may increase the prevalence of acquired cardiovascular diseases, such as VTE or $\mathrm{AF}^{26,27}$ two clinical situations in which the indication for anticoagulant therapy is indubitable. ${ }^{28,29}$ In a multicenter study from four PH centers in France, we found that twothirds of patients with precapillary $\mathrm{PH}$ were receiving anticoagulant therapy. ${ }^{30}$ of note, anticoagulant therapy was prescribed for secondary prevention of VTE or AF in one half of the patients. ${ }^{30}$ This significant proportion was subsequently confirmed in another retrospective cohort study from the Unites States and Spain in which $60.2 \%$ of PAH patients were treated with oral anticoagulants. ${ }^{31}$

\section{Patients with Congenital Heart Disease-Related PAH}

Patients with PAH and cyanotic congenital heart diseases, especially those with Eisenmenger physiology, are quite susceptible to the development of thrombosis. The main manifestation is insitu pulmonary artery thrombosis rather than PE and may be present in up to one-fifth of patients with Eisenmenger syndrome. Age was the only factor associated with pulmonary thrombosis in a cohort of 98 patients with cyanotic congenital heart disease. ${ }^{32}$ Administration of prophylactic antithrombotic therapy in these patients may lead to an increased risk of bleeding complications, which sometimes can be fatal in this population. ${ }^{33}$ Thus, the routine use of anticoagulation in patients with Eisenmenger syndrome is under debate.

\section{Patients with CTEPH}

CTEPH is defined by the presence of precapillary PH associated with persistent perfusion defects at lung perfusion scan, after at least 3 months of effective anticoagulant therapy. ${ }^{5}$ In the majority of cases, CTEPH is a complication of acute PE. Hence, anticoagulant therapy is prescribed to avoid a recurrent $\mathrm{VTE} / \mathrm{PE}$, and to prevent in situ pulmonary artery thrombosis. $^{34}$

\section{What Do the Guidelines Say?}

Every 5 years, the world's experts in PH meet to discuss scientific advances and update management proposals. This work updates the scientific knowledge in the field of PAH from experts invited by the European Society of Cardiology (ESC) and the European Respiratory Society (ERS), leading to the development of European recommendations (the latest dating from 2015). ${ }^{5}$

According to 2015 ESC/ERS guidelines, ${ }^{5}$ anticoagulant therapy may be considered in patients with idiopathic 
$\mathrm{PAH}$, heritable PAH, and PAH due to use of anorexigens (class of recommendation: IIb; level of evidence: C). For patients with CTD-PAH (like scleroderma), oral anticoagulation may be considered on an individual basis and in the presence of thrombophilic predisposition (class of recommendation: IIb; level of evidence: $C$ ).

In patients with CTEPH, lifelong anticoagulant therapy is recommended as the first step of treatment (class of recommendation: I; level of evidence: C), according to $2015 \mathrm{ESC} /$ ERS PH guidelines. ${ }^{5}$ Recently, European experts proposed an update of the scientific guidelines on acute PE. ${ }^{35}$ These guidelines included a dedicated chapter on CTEPH management, confirmed the recommendation of lifelong anticoagulation, and indicated that VKA was the recommended regimen. This recommendation is in agreement with national guidelines from European countries. ${ }^{36,37}$

Finally, in patients with congenital heart-disease-related $\mathrm{PAH}$, oral anticoagulant treatment may be considered in patients with pulmonary arterial thrombosis or signs of heart failure, in the absence of significant hemoptysis (class of recommendation: IIb; level of evidence: C). ${ }^{5}$

\section{Unmet Needs, Recent Advances, Ongoing Studies}

\section{Determining the Efficacy of Anticoagulant Therapy on the Survival of Patients with PAH}

As previously discussed, although anticoagulant therapy is recommended by the guidelines as a potential treatment of $\mathrm{PAH}$, the level of evidence is low. Putting aside the results of the ancillary analysis of a therapeutic trial performed before the appearance of specific PAH drugs, ${ }^{14}$ we have mainly epidemiological data with discordant results. A cohort study of patients with CTD-PAH (mainly scleroderma) suggested that patients who were receiving anticoagulation therapy with VKAs had better survival than those who did not receive anticoagulant therapy (-Table 1 ). ${ }^{38}$ To date, two central studies have been conducted based on national or international clinical registries: the COMPERA ${ }^{39}$ and the REVEAL ${ }^{40}$ registries (-Table 1). The COMPERA registry is an international European PAH registry ( $82 \%$ of patients coming from Germany). Among 2,414 patients with PAH, the authors analyzed the clinical evolution of 1,283 incident cases, and compared the survival of two groups: those who never received anticoagulant therapy during the 3 years of follow-up versus those who received anticoagulant therapy at any time during the follow-up. Of the whole cohort of patients with incident $\mathrm{PAH}, 58 \%$ received anticoagulant therapy (66\% idiopathic PAH subgroup, $44 \%$ other forms of $\mathrm{PAH}$ ). Patients exposed to anticoagulant therapy at any time of the follow-up were older, and had more severe diseases (more frequently with a World Health Organization functional class III/IV, a need for combination targeted PAH therapy, and worse hemodynamics). Multivariable regression analysis showed that patients with idiopathic PAH who received anticoagulant therapy had a lower risk of death (hazard ratio $[\mathrm{HR}]=0.79,95 \%$ confidence interval $[\mathrm{CI}]$ : 0.660.94). This finding was not replicated in other forms of PAH (mainly scleroderma), in whom a trend toward a lower survival was reported in patients exposed to anticoagulant therapy. This work suffers from some limitations, such as the absence of randomization, the lack of data on the prevalence of validated indication for anticoagulant therapy, and the absence of prospective monitoring of bleeding events. ${ }^{29}$

The REVEAL ${ }^{40}$ registry is an ongoing U.S. clinical registry of patients with PAH, which differs from other registries by accepting a higher threshold of pulmonary capillary wedge pressure $(\leq 18 \mathrm{~mm} \mathrm{Hg})$. As was the case with the COMPERA registry, investigators from the REVEAL registry assessed the association between chronic anticoagulation (with warfarin) and outcomes in patients with PAH. To avoid the "immortal time bias," the authors performed a sensitivity analysis on patients in whom warfarin was initiated during the study. Controls consisted of patients who were never exposed to anticoagulant therapy during the follow-up. Among the 2,197 included patients, 1,116 started warfarin before or at enrollment. Of these, 218 patients (163 idiopathic PAH patients and 55 PAH patients associated with scleroderma) started warfarin after enrollment and were compared with matched controls in the sensitivity analysis. The authors did not find any positive effect of warfarin (mean length of exposure: 1 year) in patients with idiopathic PAH. When analysis was modified to include patients who received warfarin at any time, the survival was lower in patients with PAH associated with scleroderma who received warfarin at any time of the study. Similar to the COMPERA trial, the results of the REVEAL study were suffer from lack of randomization, lack of data on the prevalence of validated indication for anticoagulant therapy, and absence of prospective monitoring of bleeding events.

These retrospective analyses of prospective epidemiological cohorts were pooled to conduct a meta-analysis which assessed the effect of anticoagulant therapy on survival in patients with $\mathrm{PAH} .{ }^{41}$ The authors concluded that anticoagulant therapy significantly reduced mortality in the overall PAH cohort ( $\mathrm{HR}=0.73,95 \% \mathrm{CI}$ : $0.57-0.93$ ), with a similar effect in patients with idiopathic $\mathrm{PAH}$, while it was associated with an increase in mortality in patients with PAH associated with scleroderma ( $\mathrm{HR}=1.58,95 \% \mathrm{CI}$ : 1.08-2.31). Besides the large number of patients and the homogeneous effect of anticoagulant therapy in the whole cohort and in patients with idiopathic PAH, the results of the meta-analysis suffer from the low quality of data, with a moderate risk of bias, as well as the absence of randomized controlled trials.

Currently, only two trials with anticoagulation drugs are ongoing in patients with $\mathrm{PAH}$, both focusing on patients with PAH associated with scleroderma. The SPHYNX trial aims to assess the efficacy, safety, and cost-effectiveness of treatment with apixaban in PAH associated with scleroderma over 3 years, in a multicenter, double-blind, placebo-controlled randomized controlled trial. ${ }^{42}$ Of note, the SPHYNX trial includes a cost-effectiveness analysis, and will be able to infirm the results of a previous medico-analytic study which suggested a positive effect of anticoagulant therapy in patients with idiopathic PAH but not PAH associated with scleroderma ${ }^{43}$ The Pragmatic Clinical Trials in Scleroderma 
Use of Anticoagulants in Patients with PH Bertoletti et al. 351

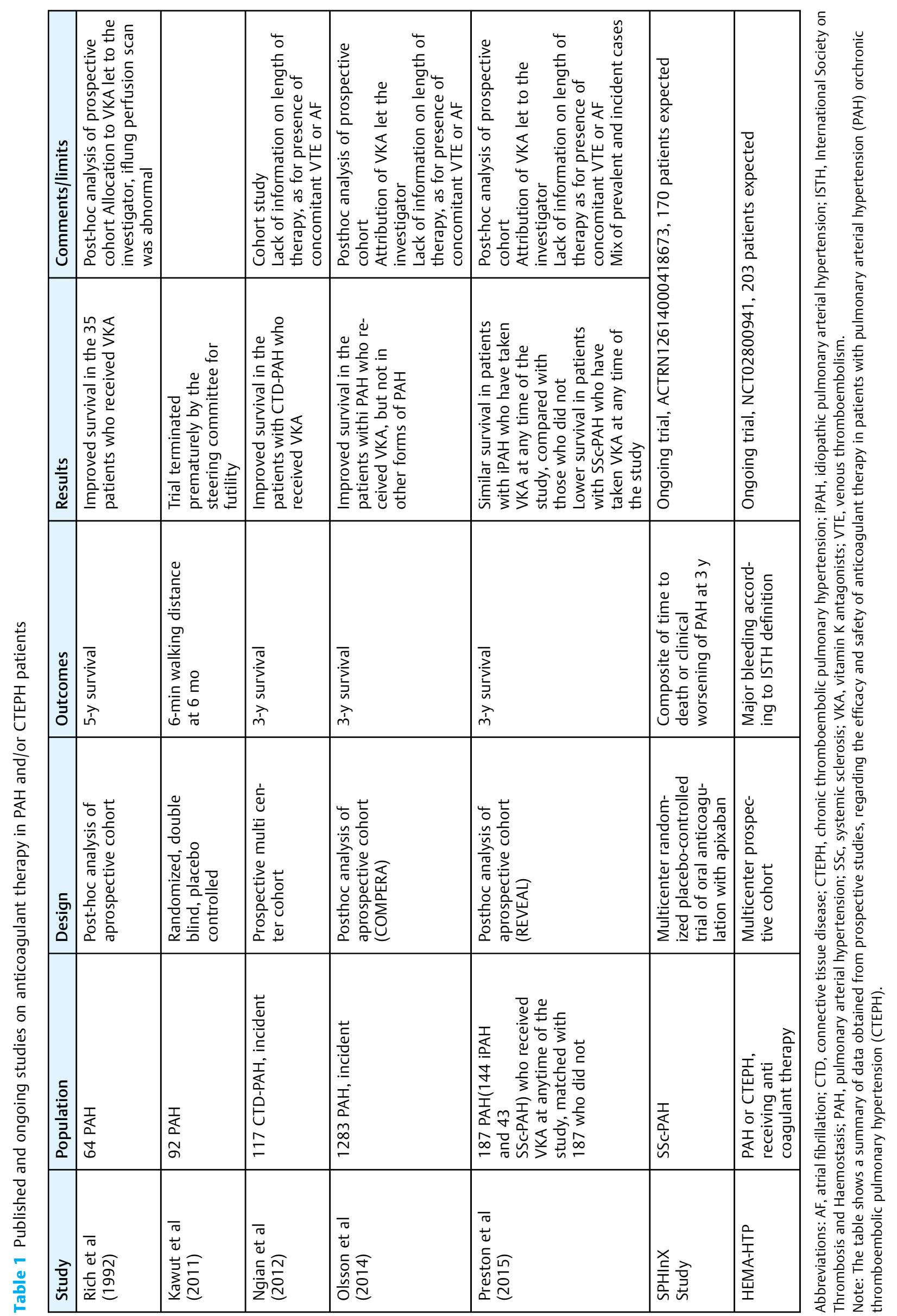


program (PCTS) is also registered on Clinicaltrial.gov (NCT03610217), and includes an arm which will evaluate the efficacy of anticoagulant therapy (warfarin, rivaroxaban, or apixaban) on PAH.

\section{Confirming the Safety of Anticoagulant Therapy in Patients with PAH or CTEPH}

The amount of available data to assess the safety of anticoagulant therapy in patients with PAH or CTEPH is less compared with that of efficacy data. This is a problem, since anticoagulant treatment increases the risk of bleeding, including life-threatening bleeding associated with PAH and CTEPH per se, such as hemoptysis, ${ }^{44}$ a situation which may particularly affect patients with PAH associated with congenital heart diseases. ${ }^{33}$

All the published data come from retrospective analyses $31,45,46$ of patients with PAH or CTEPH who received anticoagulant therapy. The first paper was a single-center retrospective study from Amsterdam. ${ }^{45}$ The authors reported the annual rate of major bleeding (defined by the International Society on Thrombosis and Haemostasis criteria $^{47}$ ) in 198 patients with precapillary $\mathrm{PH}$ (idiopathic $\mathrm{PAH}$ $[n=99]$, PAH associated with connective-tissue disease $[n=39]$, and CTEPH $[n=60])$. Of note, the international normalized ratio (INR) target range was above 2.5 and 3.5 for more than $80 \%$ of cases. Interestingly, they reported a lower rate of major bleeding in patients with CTEPH ( 2.4 for 100patient-years) than in patients with idiopathic PAH (5.4 for 100patient-years), despite CTEPH patients being older. The rate of major bleeding was markedly elevated in patients with PAH associated with connective-tissue disease (19 for 100patient-years). No factors were found to be associated with the risk of major bleeding. However, there was a significant association between the use of prostacyclin analogues as PAH-targeted therapy and major bleeding, suggesting a potential increased rate of alveolar bleeding in PAH patients with concomitant use of prostanoids and VKA reported also in a study from Japan. ${ }^{48}$ Bleeding from the gastrointestinal tract was the most frequent type of bleeding event (50\%). As with all retrospective single-center studies, the results are weakened by a center bias (as illustrated by the INR target higher than usual, as well as the age which was younger than the age of patients included in multicenter prospective registries). Roldan et al confirmed these results in a retrospective study of $201 \mathrm{PAH}$ patients, with an incidence rate for major bleeding of 4.7 per 100 patient-years (5.0 per 100 patient-years for idiopathic $\mathrm{PAH}$ ), ${ }^{31}$ but without the excess in major bleeding events for patients with CTDPAH (6.3 per 100 patient-years). Baseline factors which were associated with the occurrence of bleeding were a HAS-BLED score equal or greater than 3 , history of prior bleeding, poor anticoagulation control, and increased number of concomitant medications. ${ }^{31}$ Finally, Jujo-Sanada et al ${ }^{46}$ evaluated the risk of clinically relevant bleeding in 72 patients with CTEPH under warfarin in a retrospective single-center study. The incidence of major bleeding was to $5.0 \%$ per person-year (two times more than in the Henken et al's study), which seems to be more in agreement with the current literature in the field of unselected patients with acute VTE. ${ }^{49}$ All these three studies suffer from their retrospective design, and their single-center nature for two of them. One prospective multicenter cohort is ongoing (NCT02800941), ${ }^{50}$ which aims to estimate the incidence of major bleeding in patients with $\mathrm{PAH}$ or CTEPH receiving anticoagulant therapy, with a sufficient power also to provide some predictive factors.

\section{Tailoring the Best Anticoagulant Option for Patients with CTEPH, as well as for PAH Patients Who Have an Indication for Anticoagulation}

The emergence of DOACs deeply modified the landscape of anticoagulant therapy. ${ }^{6}$ In patients with VTE as well as in nonvalvular AF, they have demonstrated a better safety, with at least noninferior efficacy compared with warfarin. ${ }^{51-53}$ Conversely, negative results were shown in patients with a mechanical heart valve or antiphospholipid syndrome. ${ }^{54,55}$

In patients with $\mathrm{PAH}$ or CTEPH who receive VKA, there is a significant risk of pharmacological interactions, ${ }^{56-58}$ but this may be monitored with INR measurements. We also have data suggesting that the quality of anticoagulation with warfarin, as evaluated with the time in therapeutic range, is low, exposing these patients to complications. ${ }^{59}$ These points may support the role of DOACs, at least in patients with a validated indication such as secondary prevention of VTE, or AF. Basic research suggested that an anti-Xa factor (rivaroxaban) may be efficient in an experimental model of PAH. ${ }^{60}$ In the German cohort, about half of patients with CTEPH were receiving rivaroxaban for secondary VTE prevention. ${ }^{61}$ Unfortunately, no information on bleeding complications was provided. Very recently, investigators from CTEPH centers in the United Kingdom shared their experience with the use of DOACs in CTEPH patients following pulmonary endarterectomy. ${ }^{62}$ In their retrospective analysis, a similar rate of bleeding was found between patients under VKA and those under DOACs, while more recurrent VTE events were diagnosed in patients receiving DOACs. These results are striking, as available evidence in VTE studies has led to the opposite findings: a lower rate of bleeding with similar efficacy. ${ }^{63}$ This may be explained by pharmacological issues between DOACs and the targeted PAH drugs, as some of them (bosentan, phosphodiesterase-5 inhibitors, riociguat) may provide significant drug-drug interaction with DOACs. ${ }^{64-66}$ Hence, a modified bioaccumulation of DOACs in patients receiving treatment for PAH or CTEPH may be part of the absence of better safety compared with VKAs. In terms of efficacy, it has been demonstrated that VTE recurrence may be an explanation of $\mathrm{PH}$ recurrence after surgery, ${ }^{67}$ highlighting the need for a highly efficient therapy. Historically, the antiphospholipid syndrome has been recognized as a significant thrombotic risk factor of CTEPH. Its prevalence in CTEPH patients ranges from 2.5 to $7.7 \% .{ }^{68,69}$ The therapeutic impact is important, as a triple-positive antiphospholipid antibody profile is a condition in which DOACs are contraindicated, ${ }^{55}$ and patients should be prescribed VKAs. This issue has been raised as a potential explanation of reported failure of DOACs in CTEPH patients from the United Kingdom study. ${ }^{70}$ Moreover, the fact that cases were identified through the routine 
screening of asymptomatic patients as well as low adherence of treatment with DOACs might also explain this finding. However, all these results should be confirmed in future randomized trials or larger multicenter registries. In addition, the reduceddosage of DOACs does not seem a good option in patients with CTEPH.

\section{Time Capsule}

In the future ideally:

- PAH may be a curable disease, with the development of personalized therapy according to genomics information, patient symptoms, and laboratory results.

- A prospective cohort management trial studying the use of DOACs in PAH and CTEPH could be an initial step toward the understanding of the efficacy and safety of these compounds.

- Later, a randomized controlled trial with sufficient power will respond to the question of anticoagulant effect on the prognosis of patients with PAH.

- Incidence of CTEPH after a PE episode is reduced, with the development of prophylactic strategies.

- Bleeding complications will be minimized primarily by new compounds not interfering with physiological clotting and with minimal drug-drug interactions.

- Pleiotropic molecules improving inflammation and thrombogenicity will be added in the armamentarium of approved targeted therapies for PAH and CTEPH.

\section{Authors}

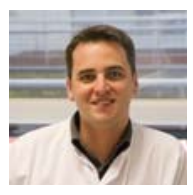

\section{Laurent Bertoletti}

Laurent Bertoletti is Medical Doctor and Professor of Medicine in the University Hospital of St-Etienne (CHUSE), France.

He graduated as a respiratory physician in the faculty of Medicine of St-Etienne, France.

After a fellowship in the department of Pr Gerald SIMONNEAU (Paris), he created and developed the pulmonary hypertension program of the University Hospital of St-Etienne.

After a post-doc in the "Angiology and Haemostasis" department of Pr H BOUNAMEAUX (Geneva, Switzerland), he moved to the Vascular Medicine department of the CHUSE, department he leads since several years.

His current researches focus on the management of vascular diseases, with a specific attention to anticoagulant therapy in patients with pulmonary vascular diseases. He is highly involved in clinical research in venous thromboembolism (via the French INNOVTE network, http://www.innovte-thrombosisnetwork.eu/fr/reseau-innovte), with interaction in basic sciences (via the INSERM U1059 SAINBIOSE, https://www. univ-st-etienne.fr/fr/sainbiose.html). He is the national coordinator of the RIETE registry (https://rieteregistry.com/). He authored more than 150 references on Pubmed (https://www. researchgate.net/profile/Laurent_Bertoletti).

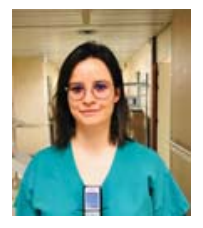

Valentine Mismetti

Valentine Mismetti is resident in respiratory medicine.

She graduated her medical school in the University Hospital of St-Etienne (CHUSE), France.

Her clinical and scientific interests focus on pulmonary vascular diseases and pleural diseases, and the pharmacology of their therapies.

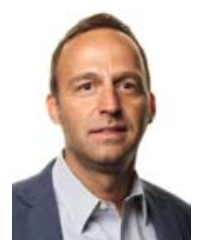

\section{George Giannakoulas}

George Giannakoulas, MD, PhD runs the Pulmonary Hypertension and Adult Congenital Heart Disease Clinics at the AHEPA Hospital in Aristotle University of Thessaloniki, Greece. He is an Assistant Professor in Cardiology. He runs numerous international clinical trials on pulmonary hypertension and heart failure. Dr Giannakoulas has spent most of his professional career in Thessaloniki, with a 2-year fellowship at the Royal Brompton Hospital in London. He is currently the National ESC Guidelines Coordinator of the Hellenic Cardiological Society, the National Coordinator for the Adult Congenital Heart Disease (CHALLENGE) registry and has served as a Member of the Nucleus of the Working Group on Pulmonary Circulation and Right Ventricular Function of the European Society of Cardiology. Dr Giannakoulas' research activities are mainly focused on heart failure, pulmonary hypertension and congenital heart disease. He has published 175 scientific publications indexed in Pubmed (5044 citations, h-factor=29, March 2020), has been a reviewer for high impact journals and serves as an Associate Editor in Hellenic Journal of Cardiology, European Journal of Preventive Cardiology, Journal of Biomedicine. In 2016, students of the Medical School of Aristotle University of Thessaloniki rated him as the second best Teacher of the Year.

\section{References}

1 Galiè N, McLaughlin VV, Rubin LJ, Simonneau G. An overview of the 6th World Symposium on Pulmonary Hypertension. Eur Respir J 2019;53(01):1802148

2 Simonneau G, Montani D, Celermajer DS, et al. Haemodynamic definitions and updated clinical classification of pulmonary hypertension. Eur Respir J 2019;53(01):1801913

3 Abenhaim L, Moride Y, Brenot F, et al; International Primary Pulmonary Hypertension Study Group. Appetite-suppressant drugs and the risk of primary pulmonary hypertension. $\mathrm{N}$ Engl J Med 1996;335(09):609-616

4 Guignabert C, Tu L, Girerd B, et al. New molecular targets of pulmonary vascular remodeling in pulmonary arterial hypertension: importance of endothelial communication. Chest 2015;147 (02):529-537

5 Galiè N, Humbert M, Vachiery J-L, et al; ESC Scientific Document Group. 2015 ESC/ERS Guidelines for the diagnosis and treatment of pulmonary hypertension: The Joint Task Force for the Diagnosis and Treatment of Pulmonary Hypertension of the European Society of Cardiology (ESC) and the European Respiratory Society (ERS): Endorsed by: Association for European Paediatric and Congenital Cardiology (AEPC), International Society for Heart and Lung Transplantation (ISHLT). Eur Heart J 2016;37(01): $67-119$ 
6 Bertoletti L, Ollier E, Duvillard C, et al. Direct oral anticoagulants: current indications and unmet needs in the treatment of venous thromboembolism. Pharmacol Res 2017;118:33-42

7 Humbert M, Guignabert C, Bonnet S, et al. Pathology and pathobiology of pulmonary hypertension: state of the art and research perspectives. Eur Respir J 2019;53(01):1801908

8 Fuster V, Steele PM, Edwards WD, Gersh BJ, McGoon MD, Frye RL. Primary pulmonary hypertension: natural history and the importance of thrombosis. Circulation 1984;70(04):580-587

9 Christ G, Graf S, Huber-Beckmann R, et al. Impairment of the plasmin activation system in primary pulmonary hypertension: evidence for gender differences. ThrombHaemost 2001;86(02): 557-562

10 Huber K, Beckmann R, Frank H, Kneussl M, Mlczoch J, Binder BR. Fibrinogen, t-PA, and PAI-1 plasma levels in patients with pulmonary hypertension. Am J Respir Crit Care Med 1994;150(04): 929-933

11 Herve P, Humbert M, Sitbon O, et al. Pathobiology of pulmonary hypertension. The role of platelets and thrombosis. Clin Chest Med 2001;22(03):451-458

12 Hoeper MM, Sosada M, Fabel H. Plasma coagulation profiles in patients with severe primary pulmonary hypertension. Eur Respir J 1998;12(06):1446-1449

13 Miniati M, Fiorillo C, Becatti M, et al. Fibrin resistance to lysis in patients with pulmonary hypertension other than thromboembolic. Am J Respir Crit Care Med 2010;181(09):992-996

14 Rich S, Kaufmann E, Levy PS. The effect of high doses of calciumchannel blockers on survival in primary pulmonary hypertension. N Engl J Med 1992;327(02):76-81

15 Frank H, Mlczoch J, Huber K, Schuster E, Gurtner HP, Kneussl M. The effect of anticoagulant therapy in primary and anorectic druginduced pulmonary hypertension. Chest 1997;112(03):714-721

16 Johnson SR, Granton JT, Mehta S. Thrombotic arteriopathy and anticoagulation in pulmonary hypertension. Chest 2006;130(02): $545-552$

17 Johnson SR, Mehta S, Granton JT. Anticoagulation in pulmonary arterial hypertension: a qualitative systematic review. Eur Respir J 2006;28(05):999-1004

18 Humbert M, Sitbon O, Chaouat A, et al. Pulmonary arterial hypertension in France: results from a national registry. Am J Respir Crit Care Med 2006;173(09):1023-1030

19 Robbins IM, Kawut SM, Yung D, et al. A study of aspirin and clopidogrel in idiopathic pulmonary arterial hypertension. Eur Respir J 2006;27(03):578-584

20 Aytekin M, Aulak KS, Haserodt S, et al. Abnormal platelet aggregation in idiopathic pulmonary arterial hypertension: role of nitric oxide. Am J Physiol Lung Cell Mol Physiol 2012;302(06): L512-L520

21 Kawut SM, Bagiella E, Lederer DJ, et al; ASA-STAT Study Group. Randomized clinical trial of aspirin and simvastatin for pulmonary arterial hypertension: ASA-STAT. Circulation 2011;123(25): 2985-2993

22 Humbert M. The burden of pulmonary hypertension. Eur Respir J 2007;30(01):1-2

23 Rich S, Dantzker DR, Ayres SM, et al. Primary pulmonary hypertension. A national prospective study. Ann Intern Med 1987;107 (02):216-223

24 Humbert M, Sitbon O, Yaïci A, et al; French Pulmonary Arterial Hypertension Network. Survival in incident and prevalent cohorts of patients with pulmonary arterial hypertension. Eur Respir J 2010;36(03):549-555

25 Benza RL, Miller DP, Gomberg-Maitland M, et al. Predicting survival in pulmonary arterial hypertension: Insights from the registry to evaluate early and long-term pulmonary arterial hypertension disease management (REVEAL). Circulation 2010; 122(02):164-172

26 Hoeper MM, Huscher D, Ghofrani HA, et al. Elderly patients diagnosed with idiopathic pulmonary arterial hypertension: results from the COMPERA registry. Int J Cardiol 2013;168(02): 871-880

27 Mercurio V, Peloquin G, Bourji KI, et al. Pulmonary arterial hypertension and atrial arrhythmias: incidence, risk factors, and clinical impact. Pulm Circ 2018;8(02):2045894018769874

28 Bertoletti L, Delavenne X, Montani D. Antithrombotics in pulmonary hypertension: more work needed before we turn to newer agents!. Eur Respir J 2013;41(04):775-777

29 Bertoletti L, Mismetti P, Decousus H. Letter by Bertoletti et al regarding article, "Anticoagulation and survival in pulmonary arterial hypertension: results from the Comparative, Prospective Registry of Newly Initiated Therapies for Pulmonary Hypertension (COMPERA)". Circulation 2014; 130(12):e108

30 Gabriel L, Delavenne X, Bedouch P, et al. Risk of direct oral anticoagulant bioaccumulation in patients with pulmonary hypertension. Respiration 2016;91(04):307-315

31 Roldan T, Rios JJ, Villamañan E, Waxman AB. Complications associated with the use of oral anticoagulation in patients with pulmonary arterial hypertension from two referral centers. Pulm Circ 2017;7(03):692-701

32 Jensen AS, Idorn L, Thomsen C, et al. Prevalence of cerebral and pulmonary thrombosis in patients with cyanotic congenital heart disease. Heart 2015;101(19):1540-1546

33 Giannakoulas G, Boutsikou M. The Gordian knot of thromboembolism in congenital heart disease. Heart 2015;101(19):1523-1524

34 Pepke-Zaba J, Ghofrani H-A, Hoeper MM. Medical management of chronic thromboembolic pulmonary hypertension. Eur Respir Rev 2017;26(143):160107

35 Konstantinides SV, Meyer G, Becattini C, et al. 2019 ESC Guidelines for the diagnosis and management of acute pulmonary embolism developed in collaboration with the European Respiratory Society (ERS). Eur Heart J 2020;41(04):543-603

36 Sanchez O, Benhamou Y, Bertoletti L, et al. Recommendations of good practice for the management of thromboembolic venous disease in adults. Short version [in French]. Rev Mal Respir 2019; 36(02):249-283

37 Grünig E, Benjamin N, Krüger U, et al. General measures and supportive therapy for pulmonary arterial hypertension: updated recommendations from the Cologne Consensus Conference 2018. Int J Cardiol 2018;272S:30-36

38 Ngian GS, Stevens W, Prior D, et al. Predictors of mortality in connective tissue disease-associated pulmonary arterial hypertension: a cohort study. Arthritis Res Ther 2012;14(05):R213

39 Olsson KM, Delcroix M, Ghofrani HA, et al. Anticoagulation and survival in pulmonary arterial hypertension: results from the Comparative, Prospective Registry of Newly Initiated Therapies for Pulmonary Hypertension (COMPERA). Circulation 2014;129 (01):57-65

40 Preston IR, Roberts KE, Miller DP, et al. Effect of warfarin treatment on survival of patients with pulmonary arterial hypertension (PAH) in the Registry to Evaluate Early and Long-Term PAH Disease Management (REVEAL). Circulation 2015;132(25):2403-2411

41 Khan MS, Usman MS, Siddiqi TJ, et al. Is anticoagulation beneficial in pulmonary arterial hypertension? Circ Cardiovasc Qual Outcomes 2018;11(09):e004757

42 Calderone A, Stevens W, Prior D, et al. Multicentrerandomised placebo-controlled trial of oral anticoagulation with apixaban in systemic sclerosis-related pulmonary arterial hypertension: the SPHInX study protocol. BMJ Open 2016;6(12):e011028

43 Jose A, Eckman MH, Elwing JM. Anticoagulation in pulmonary arterial hypertension: a decision analysis. Pulm Circ 2019;9(04): 2045894019895451

44 Żyłkowska J, Kurzyna M, Pietura R, et al. Recurrent hemoptysis: an emerging life-threatening complication in idiopathic pulmonary arterial hypertension. Chest 2011;139(03):690-693

45 Henkens IR, Hazenoot T, Boonstra A, Huisman MV, Vonk-Noordegraaf A. Major bleeding with vitamin K antagonist anticoagulants in pulmonary hypertension. Eur Respir J 2013;41(04):872-878 
46 Jujo-Sanada T, Tanabe N, Sakao S, et al. The anticoagulant effects of warfarin and the bleeding risk associated with its use in patients with chronic thromboembolic pulmonary hypertension at a specialist center in Japan: a retrospective cohort study. Pulm Circ 2017;7(03):684-691

47 Schulman S, Kearon C; Subcommittee on Control of Anticoagulation of the Scientific and Standardization Committee of the International Society on Thrombosis and Haemostasis. Definition of major bleeding in clinical investigations of antihemostatic medicinal products in non-surgical patients. J ThrombHaemost 2005;3(04):692-694

48 Ogawa A, Matsubara H, Fujio H, et al. Risk of alveolar hemorrhage in patients with primary pulmonary hypertension-anticoagulation and epoprostenol therapy. Circ J 2005;69(02):216-220

49 Nieto JA, Camara T, Gonzalez-Higueras E, et al; RIETE Investigators. Clinical outcome of patients with major bleeding after venous thromboembolism. Findings from the RIETE Registry. ThrombHaemost 2008;100(05):789-796

50 Clinicaltrials.gov. Bleeding frequency under anticoagulant treatment in pulmonary hypertension (HEMA-HTP), (NCT02800941), Available at: https://clinicaltrials.gov/ct2/show/NCT02800941? term=anticoagulant\&cond $=$ Pulmonary + Arterial + Hypertension\&draw=2\&rank=1. Published 2016. Accessed May 25, 2020

51 Mai V, Bertoletti L, Cucherat M, et al. Extended anticoagulation for the secondary prevention of venous thromboembolic events: an updated network meta-analysis. PLoS One 2019;14(04):e0214134

52 Mai V, Guay CA, Perreault L, et al. Extended anticoagulation for VTE: a systematic review and meta-analysis. Chest 2019;155(06): 1199-1216

53 López-López JA, Sterne JAC, Thom HHZ, et al. Oral anticoagulants for prevention of stroke in atrial fibrillation: systematic review, network meta-analysis, and cost effectiveness analysis. BMJ 2017; 359:j5058

54 Eikelboom JW, Connolly SJ, Brueckmann M, et al; RE-ALIGN Investigators. Dabigatran versus warfarin in patients with mechanical heart valves. N Engl J Med 2013;369(13):1206-1214

55 Pengo V, Denas G, Zoppellaro G, et al. Rivaroxaban vs warfarin in high-risk patients with antiphospholipid syndrome. Blood 2018; 132(13):1365-1371

56 Spangler ML, Saxena S. Warfarin and bosentan interaction in a patient with pulmonary hypertension secondary to bilateral pulmonary emboli. Clin Ther 2010;32(01):53-56

57 Treiber A, Schneiter R, Häusler S, Stieger B. Bosentan is a substrate of human OATP1B1 and OATP1B3: inhibition of hepatic uptake as the common mechanism of its interactions with cyclosporin A, rifampicin, and sildenafil. Drug MetabDispos 2007;35(08):1400-1407

58 Walker G, Mandagere A, Dufton C, Venitz J. The pharmacokinetics and pharmacodynamics of warfarin in combination with ambri- sentan in healthy volunteers. Br J Clin Pharmacol 2009;67(05): 527-534

59 Roldan T, Villamañán E, Rios JJ, Waxman AB. Assessment of the quality of anticoagulation management in patients with pulmonary arterial hypertension. Thromb Res 2017;160(October):83-90

60 Delbeck M, Nickel KF, Perzborn E, et al. A role for coagulation factor Xa in experimental pulmonary arterial hypertension. Cardiovasc Res 2011;92(01):159-168

61 Olsson KM, Wiedenroth CB, Kamp J-C, et al. Balloon pulmonary angioplasty for inoperable patients with chronic thromboembolic pulmonary hypertension: the initial German experience. Eur Respir J 2017;49(06):1602409

62 Bunclark K, Newnham M, Chiu YD, et al. A multicenter study of anticoagulation in operable chronic thromboembolic pulmonary hypertension. J ThrombHaemost 2020;18(01):114-122

63 Bertoletti L, Jaïs X, Bezzeghoud S, Delavenne X. Direct oral anticoagulants: Still too early for prime time after pulmonary endarteriectomy? J ThrombHaemost 2020;18(03):758-759

64 Margelidon-Cozzolino V, Hodin S, Jacqueroux E, Delézay O, Bertoletti L, Delavenne X. In vitro assessment of pharmacokinetic drug-drug interactions of direct oral anticoagulants: type 5phosphodiesterase inhibitors are inhibitors of rivaroxaban and apixaban efflux by P-glycoprotein. J Pharmacol Exp Ther 2018; 365(03):519-525

65 Jacqueroux E, Mercier C, Margelidon-Cozzolino V, Hodin S, Bertoletti L, Delavenne X. In vitro assessment of P-gp and BCRP transporters-mediated drug-drug interactions of riociguat with direct oral anticoagulants. Fundam Clin Pharmacol 2020;34(01): 109-119

66 Margelidon-Cozzolino V, Delavenne X, Catella-Chatron J, et al. Indications and potential pitfalls of anticoagulants in pulmonary hypertension: would DOACs become a better option than VKAs? Blood Rev 2019;37:100579

67 Cannon JE, Su L, Kiely DG, et al. Dynamic risk stratification of patient long-term outcome after pulmonary endarterectomy: results from the United Kingdom national cohort. Circulation 2016;133(18):1761-1771

68 Bunclark K, Ansaripour A, Sheares K, Toshner M. Response to letter to the editor: direct oral anticoagulants in thrombotic antiphospholipid syndrome associated with chronic thromboembolic pulmonary hypertension. J ThrombHaemost 2020;18(03):756-757

69 Jiang X, Du Y, Cheng CY, et al. Antiphospholipid syndrome in chronic thromboembolic pulmonary hypertension: a well-defined subgroup of patients. ThrombHaemost 2019;119(09):1403-1408

70 Porres-Aguilar M, Kahn SR, Hirsch AM. Direct oral anticoagulants in thrombotic antiphospholipid syndrome associated with chronic thromboembolic pulmonary hypertension. J ThrombHaemost 2020;18(03):755-756 